\title{
Formato de registros clínicos de enfermería quirúrgica
}

\author{
Eloísa Servín Morales, * Rosa Elvira Cano Lara, ** Esperanza Valencia Dolz, ** Laura Durán Ibarra, ** \\ María del Rocío Monroy Trevilla, *** Ma. del Rocío Chávez Alvarado,* Montserrat Castelán Flores,* \\ Ma. de Lourdes Espinoza Vásquez,** Abraham Ricardo Serralde Ramírez,* Martha Patricia Medel Calzadilla, * \\ Ma. Elena Bernal Becerra, ** Adriana Lauro Mendoza, ** Concepción Jacobo Vega, ** Ma. del Carmen Cruz Gómez **
}

\section{RESUMEN}

Introducción: Los registros clínicos de enfermería son testimonios documentales sobre actos y conductas profesionales donde queda reconocida toda la información sobre la actividad de enfermería, la cual hace referencia al paciente, su diagnóstico, tratamiento y evolución. Éstos se llevan a cabo en un nuevo formato (instrumento) que sirve como documento médico legal que cumple con la NOM-168-SSA-1998 ya que forma parte del expediente clínico; se utiliza para registrar las intervenciones que se desempeñan, apoyándose en el Proceso Enfermero; en él se registra la atención que se proporciona a la persona que va a ser intervenida, garantizando así la seguridad del paciente de acuerdo a la Cuarta Meta Internacional propuesta en la Cirugía Segura; así mismo, se registra la continuidad de los cuidados de forma racional y sistemática, favoreciendo también la calidad de la atención y siendo una prueba objetiva de los cuidados que se brindan. Se basa en la teoría de Orem. Objetivo: Analizar las necesidades de los profesionales de enfermería para realizar el diseño de un instrumento de registros clínicos de enfermería quirúrgica. Metodología: Cualitativa, apoyados en la fenomenología; se llevan a cabo reuniones de profesionales de enfermería quirúrgica turno matutino con más de 30 años de experiencia para diseñar un instrumento de registros clínicos de enfermería quirúrgica. Resultados: El grupo de expertos, al terminar el diseño del formato, lo da a evaluar a colegas que lo van a utilizar y es modificado en dos ocasiones por medio de entrevista directa; se establece su viabilidad a través de encuestas al personal del área quirúrgica. Conclusiones: El instrumento de registro clínico quirúrgico cumple con los criterios de seguridad para el paciente a través de las metas internacionales y cumple con la NOM-168-SSA-1998 del expediente clínico, permite registrar todos los cuidados que se brindan durante el transcurso de la cirugía.

Palabras clave: Registros clínicos, enfermería, cuidados, intervenciones quirúrgicas.

\section{Format of records clinical nursing}

\begin{abstract}
Introduction: Clinical records are documentary evidence on nursing actions and professional conduct, which is recognized all the information about the nursing activity, which refers to the patient their diagnosis, treatment and outcome. These are carried out in a new format (instrument) that serves as a forensic document to comply with NOM-168-SSA-1998 as part of the clinical record, is used to record the interventions play, based on the Nursing Process, it records the care provided to the person who will be wiretapped; thereby, ensuring patient safety according to the fourth goal in the proposed International Safe Surgery and record the same continuity of care rational and systematic way and to encourage the quality of care and being an objective test of care are provided. It is based on Orem's theory. Objective: Analyze the needs of nurses for the

\footnotetext{
* Enfermera Neuróloga, Licenciada en Enfermería.

** Enfermera Quirúrgica.

*** Enfermera General.
}

Correspondencia: Insurgentes Sur Núm. 3877, Col. La Fama, 14269, Delegación Tlalpan, México,_D.F.E-mail: baches_servin@hotmail.com Este artículo puede ser consultado en versión completa en http://www.medigraphic.com/enfermerianeurologica
\end{abstract}


design of an instrument of surgical nursing clinical records. Methodology: Qualitative supported by phenomenology, was conducted meetings surgical nurses morning shift with over 30 years of experts to design an instrument of surgical nursing clinical records. Results: The group of experts to complete the design of format is given to assess colleagues who will use it and amended twice by direct interview, establishing its feasibility through staff surveys surgical area. Conclusions: instrument surgical clinical record meets the criteria for patient safety through international targets and comply with NOM168-SSA-1998 from clinical files, allows you to record all care is provided during the course of surgery.

Key words: Clinical records, nursing, care, surgical interventions.

\section{MARCO CONCEPTUAL}

$\mathrm{S}$ egún la Norma Oficial Mexicana NOM-168SSA1-1998 referente al expediente clínico, la hoja de enfermería deberá elaborarse por el profesional en turno y contener: 1) Hábitus exterior. 2) Gráfica de signos vitales. 3) Administración de medicamentos. 4) Fecha, hora, cantidad y vía. 5) Procedimientos realizados. 6) Observaciones.

Son considerados los registros clínicos de enfermería como el testimonio documental sobre actos y conductas profesionales, donde queda registrada toda la información sobre la actividad de enfermería, la cual hace referencia al paciente, su diagnóstico, tratamiento y evolución.

Las fallas en la atención médica afectan a uno de cada 10 enfermos en todo el mundo, siendo la seguridad del paciente una cuestión prioritaria.

La Joint Commission on Acreditation of Health Care Organizations y la Joint Commission International fueron designadas oficialmente para desempeñar conjuntamente la función de centro colaborador de la Organización Mundial de la Salud (OMS) sobre soluciones para la seguridad del paciente en 2005.

Las metas internacionales de seguridad del paciente son una de las estrategias prioritarias evaluadas en todos aquellos hospitales y clínicas certificadas por la Joint Commission International, entidad encargada de acreditar calidad y seguridad de atención a los establecimientos que quieran someterse a los estándares internacionales.

De esta manera, la OMS ha implementado 6 metas: 1) Identificar correctamente al paciente, 2) Mejorar la comunicación efectiva, 3) Mejorar la seguridad de los medicamentos de alto riesgo, 4) Garantizar cirugías en el lugar correcto, con el procedimiento correcto y al paciente correcto, 5) Reducir el riesgo de infecciones asociadas con la atención médica y 6) Reducir el riesgo de daño al paciente por causa de caídas. El propósitos de la OMS es: promover mejoras específicas en cuanto a la seguridad del paciente, detectar áreas problemáticas dentro de la atención médica, describir soluciones para estos problemas, basadas en evidencias y en el conocimiento de expertos.
El Sistema Integral de Calidad en Salud (SICALIDAD) a través de proyectos, lineamentos e instrumentos enfocados a los usuarios, los profesionales de la salud y las organizaciones, contribuyen a elevar la calidad de los servicios y la seguridad del paciente en el Sistema Nacional de Salud. De acuerdo con el Plan Nacional de Desarrollo 2007-2012 (PND), uno de los objetivos centrales de la política pública en salud es brindar servicios de salud eficientes con calidad y seguridad para el paciente.

Así mismo, se consideró la Norma Oficial Mexicana NOM168-SSAA1-1998 en este formato, ya que se integra al expediente clínico como documento legal, el cual refleja la atención medico-asistencial del paciente. Cabe destacar que la ausencia de registros de la atención al paciente puede entenderse como un incumplimiento legal, ético y profesional poniendo en duda el ejercicio de la profesión.

Por lo antes citado, se debe contar con un instrumento de registro clínico de enfermería que cumpla con la normativa oficial e integre datos que promuevan las metas internacionales de seguridad del paciente planteadas por la OMS, el cual debe ser de fácil llenado, registrando el proceso de atención de enfermería (PAE) en el área quirúrgica.

La OMS establece seis metas; éstas quedan implícitas en el formato propuesto:

Primera meta. Identificar correctamente al paciente: En el quirófano se reciben pacientes con deterioro del estado neurológico, alteraciones sensoriales y motoras que pueden llegar a crear errores de identificación adecuada del paciente; para evitar esto se exigen al menos dos formas para identificar a cada enfermo, con nombre y registro.

Segunda meta. Mejorar la comunicación efectiva: Ésta se realiza en quirófano cuando recibimos por teléfono los resultados de muestras de patología; éstos son anotados en forma completa y posteriormente se vuelven a leer por el receptor.

Tercera meta. Mejorar la seguridad de los medicamentos de alto riesgo: Es fundamental que en el instrumento de registros clínicos de enfermería quirúrgica, se registre si la premedicación anestésica se llevó a cabo o no fue necesaria para disminuir el estrés quirúrgico. El uso de fármacos antes 
y durante el acto operatorio queda sujeto a las necesidades de cada enfermo por el diagnóstico y tiempo de cirugía; los medicamentos de alto riesgo están separados y se identifican por medio de etiquetas rojas; éstos son manejados por una enfermera que asiste a los neuroanestesiólogos.

Cuarta meta. Garantizar cirugías en lugar correcto, con el procedimiento correcto y al paciente correcto: Basados en el listado de verificación quirúrgica, el cual considera el tipo de intervención quirúrgica, y el área que se va a intervenir en el pre, trans y postoperatorio, aquí se considera la NOM 170-SSA-1998, la cual establece requisitos indispensables para que el paciente sea sometido a cirugía.

Quinta meta. Reducir el riesgo de infecciones asociadas con la atención médica: El personal deberá conocer y aplicar el correcto lavado de manos, portar el uniforme adecuado para el área quirúrgica, verificar las medidas de asepsia y antisepsia.

Sexta meta. Reducir el riesgo de daño al paciente por causa de caídas: Es muy importante que al ingresar el paciente al área quirúrgica se valore si presenta riesgo de caída; si es positivo, el paciente deberá traer un brazalete de identificación.

\section{PLANTEAMIENTO DEL PROBLEMA}

Ante la carencia de un documento específico de registros clínicos de enfermería en el área quirúrgica, surge la necesidad de diseñar un instrumento en el que se registre y se informe con claridad todo lo relacionado con la intervención que llevan a cabo los profesionales de enfermería de áreas quirúrgicas con el paciente. Es importante que en su diseño se brinde seguridad al paciente de acuerdo a las metas internacionales, Norma Oficial Mexicana NOM-168SSA1-198. Se evidencia esta inquietud y se reúne un grupo de expertos para realizar el diseño; de todo esto surge la siguiente pregunta de investigación.

\section{PREGUNTA DE INVESTIGACIÓN}

¿Cómo diseñar y construir un instrumento de registros clínicos de enfermería quirúrgica?

\section{JUSTIFICACIÓN}

El profesional de enfermería del INNN que labora en el área de quirófano, carecía de un instrumento clínico efectivo donde evidenciara documentalmente las intervenciones que en esa área se realizan, teniendo que utilizar los registros de enfermería del área de hospitalización, con los limitantes de registrar las propias actividades, como lo indica la
Norma Oficial Mexicana NOM-168-SSA1-1998. Se manifiesta esta necesidad entre los profesionales quirúrgicos de mayor antigüedad quienes se dan a la tarea de investigar si existen instrumentos específicos; al no encontrar ninguna documentación que justifique sus actividades, se dan a la tarea de diseñarlo con los elementos necesarios que cumplan con la normativa vigente de acuerdo a la NOM 168, metas internacionales y teorías de enfermería para contribuir a la calidad de la atención que se proporciona en los servicios de neurocirugía, quirófano y recuperación, favoreciendo la seguridad del paciente.

\section{OBJETIVOS GENERALES}

Analizar las necesidades de los profesionales de enfermería para realizar el diseño de un instrumento de registros clínicos de enfermería quirúrgica.

\section{OBJETIVOS ESPECÍFICOS}

1) Diseñar y construir un instrumento de registros clínicos de enfermería quirúrgica.

2) Verificar la viabilidad del instrumento de registros clínicos de enfermería quirúrgica.

3) Implementar el instrumento de registros clínicos de enfermería.

\section{METODOLOGÍA}

Cualitativa, apoyada de la técnica fenomenológica, interpretativa.

\section{Procedimiento}

$1^{a}$ Etapa. El personal del área quirúrgica del turno matutino identificó la carencia de un instrumento de registros clínicos de enfermería, por lo que en consenso analizaron la necesidad de diseñar e iniciaron su construcción, de acuerdo con las necesidades de los profesionales de enfermería que lo van a utilizar.

$2^{\text {a }}$ Etapa. Cada integrante del equipo quirúrgico del turno matutino se dio a la tarea de investigar la NOM 168, Metas Internacionales, y todos aquellos elementos, que cumplan con los requisitos quirúrgicos para enriquecer el diseño de los registros clínicos de enfermería quirúrgica y sean herramientas útiles, eficientes y que agilicen el registro clínico para favorecer la seguridad del paciente.

$3^{\text {a }}$ Etapa. Interpretación superficial: Recolección de información, se planeó el diseño del instrumento, con el consenso de expertos de más de 30 años de servicio en que también consideraron la teoría de Dorotea Orem. 
Interpretación profunda. Una vez terminada la recolección de información y hechas las correcciones sugeridas y pertinentes, se creó un primer instrumento de registros clínicos de enfermería quirúrgica, tras un proceso cuidadoso de investigación, favorecido por la experiencia de los participantes.

También se elaboró un instructivo para la correcta utilización del instrumento de registros clínicos de enfermería quirúrgica (IRCEQ). Cuando se concluyo éste, se capacitó al personal de los diferentes turnos del área quirúrgica para poder aplicar e implementar este nuevo formato (instrumento IRCEQ). Así mismo, se estableció la viabilidad del instrumento de registros clínicos de enfermería quirúrgica durante 3 meses; posteriormente se realizó una evaluación en todos los turnos que laboran en el área quirúrgica, por medio de entrevista directa y la aplicación de un instrumento de encuesta.

Se analizaron los resultados y se consideraron las sugerencias, realizando las modificaciones pertinentes al instrumento; fue necesario establecer con las modificaciones una segunda verificación para conocer su viabilidad y consistencia interna, en la cual ya no se sugirió agregar o quitar ninguno de los elementos que lo integran.

Se presentó como Producto Final un Instrumento de registro clínico de enfermería quirúrgica validado por expertos de enfermería del área quirúrgica, el cual se presenta ante el Comité de Investigación.

Muestra. A conveniencia de 20 profesionales de enfermería de quirófano considerándose $100 \%$ del total de la población.

Criterios de inclusión. Se consideraron a todos los profesionales de enfermería que laboran en el área de quirófano.

Criterios de exclusión. Se excluyeron a los profesionales de enfermería del área de hospitalización.

Criterios de eliminación. Todos los profesionales de enfermería de consulta externa y servicios de diagnóstico clínico, de gabinete y laboratorio.

Resultados. Se presentó finalmente el formato de registro clínico de enfermería quirúrgica después de un proceso de seis meses con el siguiente contenido:

I. Datos generales. Existen políticas internacionales dirigidas por la OMS que exigen al menos dos formas de identificar a un paciente entre las que se encuentran: Nombre del paciente, Número de registro, Fecha de nacimiento, Pulsera con datos de identificación del paciente. En el quirófano del INNN debido a su especialidad, se reciben pacientes desorientados con deterioro del estado neurológico, alteraciones sensoriales y motoras como es la afasia u otras situaciones que pueden generar errores en la identificación correcta del paciente.
El instrumento cuenta con las cuatro formas de identificación que propone la OMS.

Nombre del agente de cuidados dependientes:

\begin{tabular}{llll}
\hline Fecha de nacimiento: $1 /$ / Edad & Sexo. F/M & Registro \\
Peso: & & \\
Folio: & & \\
Identificación de brazalete: & Sí / No & Color & \\
Cama: & Servicio: \\
Diagnóstico preoperatorio: & & \\
Cirugía programada: Sí/No & Urgencia: Sí/No & \\
\hline
\end{tabular}

El uso de indicadores dentro del Instituto, basados en los nuevos estándares internacionales de certificación recomienda: 1) Preguntar directamente a los pacientes: Nombre completo, fecha de nacimiento, verificar el brazalete de identificación blanco: sólo identificación, azul: riesgo de caídas, rojo: alergias.

Con los datos que reúne el instrumento, se cumple con la primera Meta Internacional de Seguridad del paciente, lo que contribuye a evitar caídas.

Las caídas constituyen un factor importante de las lesiones sufridas en los pacientes en hospitalización, en el quirófano su cuidado depende del personal de salud, en el registro se implementó un apartado donde se registra si el paciente presenta riesgo de caída y si es positivo se valora con la escala de Dawton.

El objetivo de la Meta VI es reducir el riesgo de daño al paciente por causas de caída.

Paciente con riesgo de caída: Sí / No Escala Downton:_-_-_-_-_-

Siendo parte fundamental del instrumento de registros clínicos de enfermería quirúrgica, la premedicación anestésica, es necesaria para disminuir el estrés quirúrgico.

\section{PREMEDICACIÓN ANESTÉSICA}

Existe un apartado donde se registran los factores de riesgo, éstos nos aportan datos para planificar los cuidados.

\section{Factores de riesgo:}

Profilaxis prequirúrgica, una de las causas más importantes de la morbilidad hospitalaria es la infección hospitalaria posterior a cirugía, la profilaxis disminuye en forma significativa infecciones de herida quirúrgica, además está sustentada por la $\mathbf{V}$ Meta internacional. 


\section{PROFILAXIS PREQUIRÚRGICA}

\section{Valoración}

\begin{tabular}{lll}
\hline Ingreso & & \\
Hora: & Fecha: / / & $\begin{array}{c}\text { Actividad } \\
\text { motora }\end{array}$ \\
Estado de conciencia: & Retraso: & \\
No de sala: & & \\
Vías venosas: & & \\
Alergias: & & \\
Lesiones dérmicas: & \\
Hora de inicio de anestesia: & \\
Hora de inicio de cirugía: & \\
Tipo de anestesia: & \\
Intubación: & \\
Abordaje: & \\
Posición: & \\
Cirugía planeada: & \\
\hline
\end{tabular}

\section{Protocolo de ingreso}

\section{Documentación oficial}

Según la Norma Oficial 168-SSA-1998 y 170-SSA-1998 los requisitos indispensables para que el paciente sea sometido a cirugía se enlistan en la hoja, se incorpora la hoja de cirugía segura para garantizar la IV meta.

\begin{tabular}{l}
\hline Documentación: \\
\hline Consentimiento informado \\
Autorización de anestesia \\
Nota preoperatoria \\
Nota preanestésica \\
Hoja de cirugía segura \\
Indicaciones médicas \\
Hoja de registros clínicos de enfermería \\
Hoja de invasivos \\
Hoja de transfusión sanguínea \\
Estudios de gabinete y resultados de laboratorio \\
Electrocardiograma \\
\hline Preparación física: \\
\hline Ayuno \\
Tricotomía quirúrgica \\
Shampoo preoperatorio \\
Baño antiséptico \\
Aseo bucal \\
Uñas sin esmalte y cortas \\
Lleva anillos, aretes, pulsera \\
Prótesis movibles \\
Drenajes y sondas \\
\hline
\end{tabular}

\section{Preparación física}

El paciente que será sometido a cirugía debe estar físicamente preparado, de acuerdo con el protocolo de ingreso y basado en las metas internacionales IV y V.

\section{Diagnóstico, planeación y ejecución}

\section{Registro de signos vitales}

La norma oficial 168-SSA-1997 establece, que es responsabilidad de la enfermera medir y graficar los signos vitales según la frecuencia establecida ya que éstos reflejan el estado de salud del paciente, así como el registro de la saturación de oxígeno y pupila (estas últimas se registran al ingreso y egreso del paciente ya que durante la cirugía se mantienen ocluidos los ojos).

\begin{tabular}{|c|c|c|c|c|c|c|}
\hline Hora & FC & $\mathrm{FR}$ & T/A & Temp & $\mathrm{SAT} \mathrm{O}_{2}$ & Pupilas \\
\hline
\end{tabular}

Para reducir eventos adversos, la Alianza Mundial por la Seguridad del Paciente identificó una serie de controles de seguridad,

\section{a) Salida (antes de que el paciente salga del quirófano)}

- Revisión visual: procedimiento realizado, recuento de gasas e instrumental

Este procedimiento se realiza en conjunto enfermera instrumentista y circulante al inicio y final de cirugía y si ésta no concluye en un turno se entrega al equipo quirúrgico entrante.

\begin{tabular}{llll}
\hline $\begin{array}{l}\text { Cuenta de } \\
\text { gasas inicio }\end{array}$ & $\begin{array}{l}\text { Dentro } \\
\text { del campo }\end{array}$ & $\begin{array}{l}\text { Fuera } \\
\text { del campo }\end{array}$ & $\begin{array}{l}\text { Conteo final } \\
\text { del turno }\end{array}$ \\
\hline $\begin{array}{l}\text { Cuenta } \\
\text { de cotomoides }\end{array}$ & & \\
$\begin{array}{l}\text { Cuenta } \\
\text { de compresas }\end{array}$ & & \\
\hline
\end{tabular}

El uso de antisépticos es parte de las prácticas que se realizan en los procedimientos quirúrgicos, para lograr el barrido de la flora transitoria y disminución de proliferación de la flora residente de la piel.

\section{Antiséptico utilizado}

El uso de fármacos en el campo operatorio queda sujeto a las necesidades del acto quirúrgico.

\section{Fármacos utilizados en campo operatorio}

La placa de electrocauterio es un dispositivo de seguridad que se fija a la piel del paciente; el personal de enfermería es 
el responsable de colocar y verificar que no genere daño, por

lo tanto debe quedar registrado el sitio donde es colocado.

\section{Placa de electrocauterio}

El registro del tipo de soluciones que se están ministrando al paciente facilita reconocer el estado hemodinámico del enfermo.

\section{Soluciones}

A

B

C

\section{Evaluación}

En el INNYN los registros clínicos de enfermería se basan en la Teoría de Dorothea Orem.

Mantenimiento de un aporte suficiente de aire

Mantenimiento de un aporte suficiente de agua

Mantenimiento de un aporte suficiente de alimentos

Provisión de los cuidados asociados con los procesos de eliminación y

los excrementos

Mantenimiento del equilibrio entre la actividad y el reposo

Mantenimiento del equilibrio entre la sociedad y la interacción social

Promoción del funcionamiento y desarrollo humano

Prevención de peligros para la vida, el funcionamiento humano y el

bienestar humano

Actividades interdependientes: Registro de medicamentos ministrados durante el procedimiento quirúrgico con especial cuidado a medicamentos de alto riesgo, según la III meta.

Actividades interdependientes medicamentos

\begin{tabular}{llll}
\hline Nombre genérico & Dosis & Vía & Horario \\
\hline
\end{tabular}

- Uso de hemostáticos e implantes

Hemostáticos e implantes utilizados
- Instalación de drenajes, líneas venosas y arteriales

Línea venosa:

Línea arterial:

Drenajes

Notas de enfermería. Es el testimonio documental sobre actos y conductas profesionales donde queda registrada toda la información referente al paciente quirúrgico. La NOM168-SSA-1 señala que el profesional de enfermería deberá realizar los registros y certificaciones correspondientes a sus intervenciones.

Notas de enfermería

\section{Término de cirugía}

\begin{tabular}{lll}
\hline Término de cirugía & Egreso & $\begin{array}{l}\text { Balance de líquidos } \\
\text { Ingreso }\end{array}$ \\
\hline Cirugía realizada: & Hora: & Soluciones: \\
& Servicio: & Medicamentos: \\
Hora de término de cirugía: & Cama: & Hemoderivados: \\
Hora de término de anestesia: & Estado de conciencia: & Total: \\
Balance total: & & \\
Control radiológico postquirúrgico: & & Uresis: \\
\hline
\end{tabular}

Cambio de instrumentista y circulante. Es importante registrar el cambio del equipo quirúrgico que se realiza al término de cada turno; conviene escribir en el expediente cuáles son las limitantes de personal. Si no es posible la resolución de la carencia se deja constancia por escrito, de esta manera se establecen los límites de la responsabilidad del personal de salud. 


\begin{tabular}{llllll}
\hline \multicolumn{2}{l}{ Cambio de instrumentista: } & & \multicolumn{3}{l}{ Cambio de circulante: } \\
\hline Sí: & No: & Hora: & Sí: & No: & Hora: \\
\hline
\end{tabular}

Agencia de cuidados. En el instrumento de registros clínicos de enfermería se documenta la participación de todo el equipo quirúrgico.

Agencia de cuidados terapéuticos

\begin{tabular}{|c|c|}
\hline Neurocirujano: & Enf. Instrumentista: \\
\hline Residente: & Enf. Circulante: \\
\hline Neuroanestesiólogo: & Enf. de microcirugía: \\
\hline Residente: & Enf. de anestesia: \\
\hline
\end{tabular}

\section{FIRMA DE JEFE DE PISO O SUPERVISORA}

\section{ANÁLISIS Y DISCUSIÓN}

Se analizaron las propuestas de cada integrante que participó para su diseño en el que se construyó el formato de registros clínicos de enfermería quirúrgica de acuerdo a la NOM-168 y NOM-170 que dan cuentan de los requisitos indispensables que debe contener un documento médicolegal que se integra en el expediente clínico de cada enfermo que es intervenido; este formato consideró las metas internacionales propuestas por la OMS. Lo que contribuye a la seguridad al paciente, considerando que lo registrado da respaldo legal al profesional de enfermería y también plasma las intervenciones llevadas a cabo en el pre, trans y postoperatorio, lo cual proporciona seguridad al paciente y favorece la calidad en la atención.

\section{CONCLUSIONES}

Se realizó el formato basándose en la experiencia de los expertos de enfermería de áreas neuroquirúrgicas del INNN, con la finalidad de facilitar los registros del profesional de enfermería, sobre todo que es una herramienta útil que se adapta a las actividades específicas en el pre, trans y postoperatorio.

El formato de registro de enfermería quirúrgica cumple con la Norma Oficial y facilita el trabajo al momento de registrar, adopta criterios de seguridad para el paciente, $y$ sin duda brinda una mejor atención, al momento de registrar la atención con cada enfermo favoreciendo la relación enfermera-paciente y el trato digno a cada enfermo, agilizando las actividades que se realizan y que antes no se podían registrar ya que algunas se omitían. El formato de registros clínico-quirúrgicos funciona como una guía para la continuidad de los cuidados en el área de recuperación. A partir de la creación de este formato se han generado nuevas líneas de investigación que se están considerando a futuro. Con la siguiente reflexión se concluye este trabajo: "A cuántas enfermeras escuchamos la tan trillada frase de «la peor enemiga de la enfermera es la propia enfermera» y con gran alegría se comenta en esta ocasión que no fue así, ya que desde que inició el proyecto para el diseño y construcción del formato unió a un grupo de profesionales del turno matutino para crearlo; conscientes de todo esto nos dimos a la tarea de reunirnos y recopilar la información necesaria para lograr este objetivo; no fuimos una, no fuimos unas, fuimos todas... dándonos cuenta que esta experiencia nos hace más fuertes, mostrando un equipo de trabajo unido que logró su meta propuesta: concluir el formato e implementarlo en el área quirúrgica."

Agradecemos infinitamente a las compañeras del área quirúrgica de los diferentes turnos; a la M. en Admon. E. Patricia Zamora Ruiz y MEE. A María Guadalupe Nava Galán se le admira y respeta; a Jesús Vallarta, mil gracias por el apoyo a este proyecto.

\section{BIBLIOGRAFÍA}

1. García-Ramírez S, Navío-Marco AM, Valentín-Morganizo L. "Nure investigación". Núm. 28 mayo-junio 2007. Madrid, España, p: 1-8 consulta 15 de abril 2012 20:00 h.

2. Muñoz GJC, Gangoso FA. "Seguridad del paciente y gestión de riesgos sanitarios". Revista de la Sociedad Madrileña de Medicina de Familia y Comunitaria. febrero 2009; 10 (3): 39. Disponible en http://www. somamfyc.com Consultado abril $15201218 \mathrm{~h}$.

3. Hospital de Costa España. "Protocolo de contención y prevención de caídas". Octubre 2002: 9.

4. http://www.manciaorg.org/foro/articulos/37282-metas- Internacionales -de Seguridad paciente.html Consultado 28 de abril 2012 a las 18:00 h.

5. Vega B. "La entrevista con los familiares del paciente neuroquirúrgico". Camagüey, Cuba, Servicios de Neurocirugía, Hospital Manuel Ascunce Domenech 2004300512 19;20 disponible www.revistaNeuroquirurgica .com/artics/v15m4/9.pdf

6. Judez GJ. "Consentimiento informado". Medicina Clínica. 2001. 14 de febrero del $201216 \mathrm{hrs}$.http://tauli.cat/tauliprofesionals p. 99-10.

7. Córdova-Ávila M, Nieto-González L . "Reflexiones del marco jurídico en las áreas quirúrgicas". Revista de la CONAMED 2011; 16 (Supl I): 23 disponible http://www.conamed.gob.mx Consultado 17 mayo 18:00.

8. "Práctica de anestesiología". Enfermería Neurológica 2011; 10 (2): 10.

9. Ortiz CO, Chávez M. "El registro de enfermería como parte del cuidado". Disponible http://facultadsalud.unicauca.edu marzo Consultado 30/5/2012 a las 8:44.

10. Cortez E. Universidad Valparaiso. Apuntes de asepsia y antisepsia 2008. Disponible en: htt://docenciaen enfermería.blogspot.mx/2008/05/ apuntes-de-asepsia-y-antisepsia-2008.htmlconsultado 3/5/2012 9:45

11. Magaña-Morales. "Consentimiento informado". Disponible Abioes.org/ files66pdf Consultado 8 de mayo del 2012 23:30 h.

12. Vázquez-Ferreyra R. "El consentimiento informado en la práctica médica”. Consulta 2012 mayo 8 disponible: http//ww:sidome.org/doctrina/ articulo/cirauf.pdf

13. Rosales S, Reyes E. "Fundamentos de enfermería". Edit. Manual Moderno. 3ra edición, p: 338. 